\title{
Research on Cultivated Land Change Based on GIS and High Resolution Remote Sensing Images
}

\author{
Haibo $\mathrm{Yu}$ \\ Ocean University of China, Qingdao, Shandong, 266100 China
}

\begin{abstract}
This paper study an automatic monitoring method for land change based on high resolution remote sensing images and GIS data, and we use three classification methods to classify and fuse the research area. Secondly, the paper calculates the corresponding map class components and compares them with their historical attributes; it can automatically monitor land use change. The experimental results show that the fuzzy decision fusion classification can significantly improve the classification effect, and it can accurately determine the change area accurately and automatically. However, there are some partial errors in the region.
\end{abstract}

Keywords: Remote sensing image; GIS database; Fuzzy classification; Cultivated land.

\section{Introduction}

The sustainable utilization of cultivated land resources is a key factor to maintain regional sustainable development, and its core is to ensure the dynamic balance of the total amount of cultivated land in the region. The state needs to set up an early warning system for cultivated land quality, which can forecast the quality of cultivated land and dynamically monitor the quality of cultivated land, so as to avoid serious deterioration of the quality of cultivated land.

Image segmentation and GIS data analysis methods are important research contents in land use change monitoring ${ }^{[1]}$. Using the image segmentation analysis method, we first use the image segmentation algorithm to obtain maps of different scales, and then use the map spots as the basic analysis object to monitor and analyze the land use change $[2,3]$. The research results show that this type of method achieves higher precision and interpretation results. We use GIS and high-resolution remote sensing imagery for land use change monitoring methods. It combines land use GIS thematic data in a specific historical period with current high-resolution remote sensing images; uses GIS vector data, directly cuts images, and then analyzes remote sensing images. The paper studies the current status of land use in the current period and automatically updates the GIS topic data ${ }^{[4-5]}$

\section{Technical route and data source}

The paper use a high-resolution remote sensing image and historical land thematic vector data, we have studied a fully automatic land dynamic monitoring method. Its general idea is: We first take the single map polygon in the GIS thematic data as the basic analysis object, and automatically obtain the sample information of the remote sensing classification; Then we carry out multi-scale segmentation for the high-resolution remote sensing images, and classify the obtained segmentation maps by object-oriented multi-classifier fuzzy decision fusion; then compare and analyze the remote sensing classification results and GIS thematic data, and automatically determine the individual patches in the GIS data. Whether there is a change in the type of land cover, and finally the automatic updating of land use information.

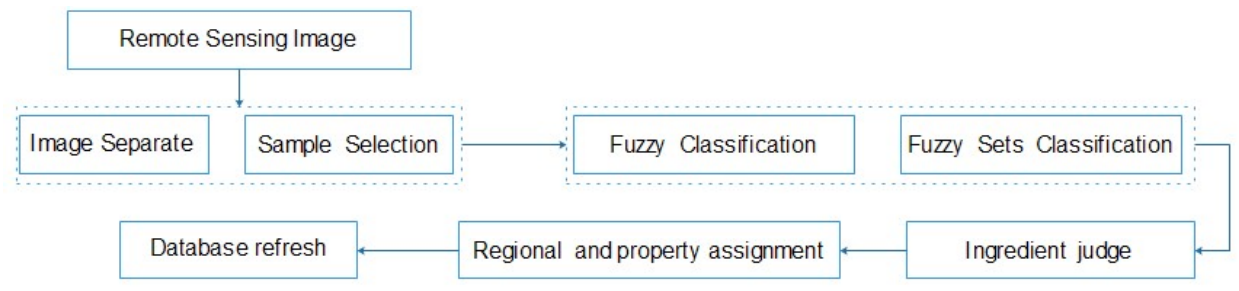

Figure 1. Flow chart of land dynamic detecting system. 
The specific implementation of this study includes automatic selection of samples, multi-scale image segmentation, fuzzy classification of remote sensing images, multi classifier combination classification, change percentage calculation, change region determination, change map spot automatic detection and so on. The flowchart is shown in Figure 1.

\section{Research on fuzzy decision of multiple classifiers}

1) Take the fuzzy classification result of each classifier as input

2) Calculate the ambiguity of each classifier separately.

3) According to the degree of fuzziness, the new class membership degree is obtained by weighting.

4) According to the classification degree of each classifier, the global weight is given to all kinds of membership.

5) The category is obtained using the principle of maximum membership.

Remote sensing image is $X=\left\{x^{1}, x^{2}, \ldots, x^{n}\right\}$, we want to use $\mathrm{n}$ feature vectors $x^{j}=\left(x_{1}^{j}, x_{2}^{j}, \ldots, x_{n}^{j}\right)$ that divided into $\mathrm{C}$ classes: $w=\left\{w_{1}, w_{2}, \ldots, w_{c}\right\}$. We use $\mathrm{m}$ classifiers to classify images. For feature pixel $\mathrm{x}$, the result of the first classifier is $\left\{u_{i}^{l}(x), u_{i}^{2}(x), \ldots, u_{i}^{c}(x)\right\}$, where $u_{i}^{j}(x) \in[0,1], j=\left[w_{1}, w_{2}, \ldots, w_{c}\right]$. The m sets fuzzy sets of pixel $\mathrm{x}$ whose input data sets can be expressed as follows.

$$
U=\left[\begin{array}{cccc}
u_{1}^{1}(x) & u_{2}^{1}(x) & \cdots & u_{m}^{1}(x) \\
u_{1}^{2}(x) & u_{2}^{2}(x) & \cdots & u_{1}^{2}(x) \\
\cdots & \cdots & \cdots & \cdots \\
u_{1}^{c}(x) & u_{2}^{c}(x) & \cdots & u_{m}^{c}(x)
\end{array}\right]
$$

Degree of membership $u_{i}^{j}(x)$ is determining value in formula (1), then set:

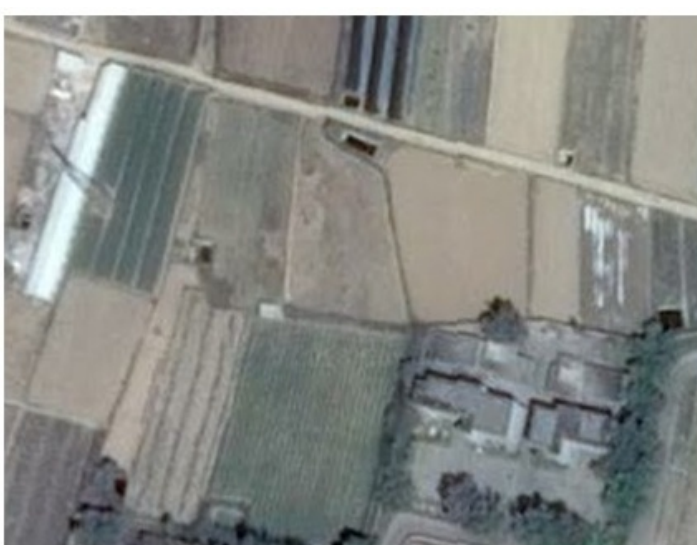

(a) Original image $\theta_{i j}=\left(u_{i}^{0}-u_{i}^{j}\right) /\left(u_{i}^{\max }-u_{i}^{\min }\right) \quad \mathrm{i}=1,2, \ldots, \mathrm{m} ; \mathrm{j}=1,2, \ldots, \mathrm{c}$

So, the $\theta_{i j}$ can be consist of a fuzzy matrix:

$$
\Delta=\left\{\begin{array}{cccc}
\theta_{11} & \theta_{12} & \cdots & \theta_{1 c} \\
\theta_{21} & \theta_{22} & \cdots & \theta_{2 c} \\
\cdots & \cdots & \cdots & \cdots \\
\theta_{m 1} & \theta_{m 2} & \cdots & \theta_{m c}
\end{array}\right\}
$$

Using the Delphi method, assessment of the impact factors of weight coefficients wi, determine the factors important degree of fuzzy set:

$$
\begin{gathered}
w=\left(w_{1}, w_{2}, \ldots, w_{m}\right), w_{i}=\sum_{j=1}^{m} u_{i}^{j} / \sum_{j=1}^{m} \sum_{i=1}^{c} u_{j}^{i} \\
d_{j}=\frac{1}{\bar{w}}\left(\sum_{i=1}^{m}\left(w_{i} \bullet \theta_{i j}\right)^{2}\right)^{0.5}
\end{gathered}
$$

Where: Where: $\bar{w}=\frac{1}{m} \sum_{i=1}^{m} w_{i}$.

When $\quad d_{i}=d_{i}\left(u_{i}, u^{0}\right)=\min \left\{d_{1}, d_{2}, \ldots, d_{n}\right\}$ classifier $i$ is the optimal classifier. After the classifier I is determined as the optimal classifier, the classification of pattern $\mathrm{X}$ is determined according to the principle of maximum membership degree.

\section{Experiment and analysis}

The experimental data source is the QuickBird image of a region in 2010. Spatial resolution: 1m (red, green, blue 3 bands), area size: $2000 \times 2000$ pixels (true color image). The experimental image area mainly includes pits, rivers, farmland, buildings, roads, bare land and many other types of features.

The experimental area has channels, ponds, bare land, orchards, vegetation, and forests. We classify remote sensing images using Mahalanobis distance, vector machine, and neural network classification methods.

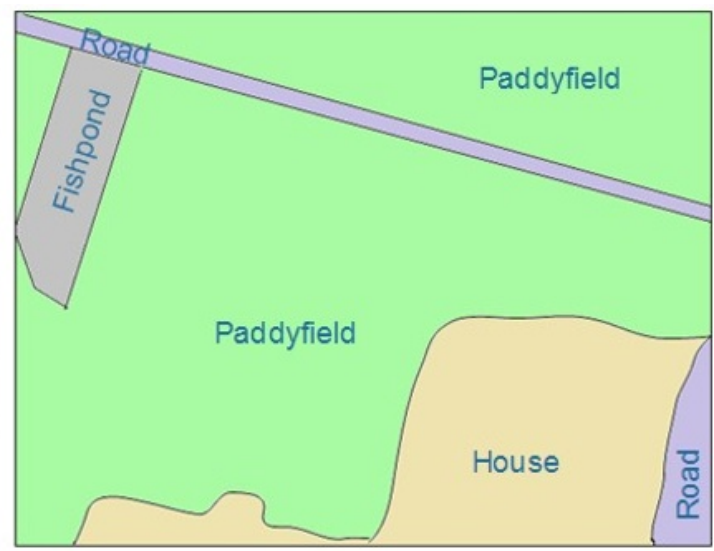

(b) Historical land GIS data

Figure 2. Remotely sensed image and GIS data of the experiment area. 
Experimental parameter settings: spectral information weight $=0.6$, shape information weight $=0.4$, segmentation scale $=(50,100,300)$, local region segmentation results are shown in Figure 3.

Experimental results:

(1) When the segmentation scale is 50 , the generated patch area is small, and the same farmland is easily divided into a plurality of different regions.

(2) When the division scale is 100 , the farmland is basically divided into independent areas, and each house in the residential area can also be divided into a single unit.

(3) When the division scale is 300 , a plurality of farmland are divided into one area, and at the same time, the phenomenon that adjacent houses are divided into one unit appears. is 100 .

This paper uses the segmentation result when the scale
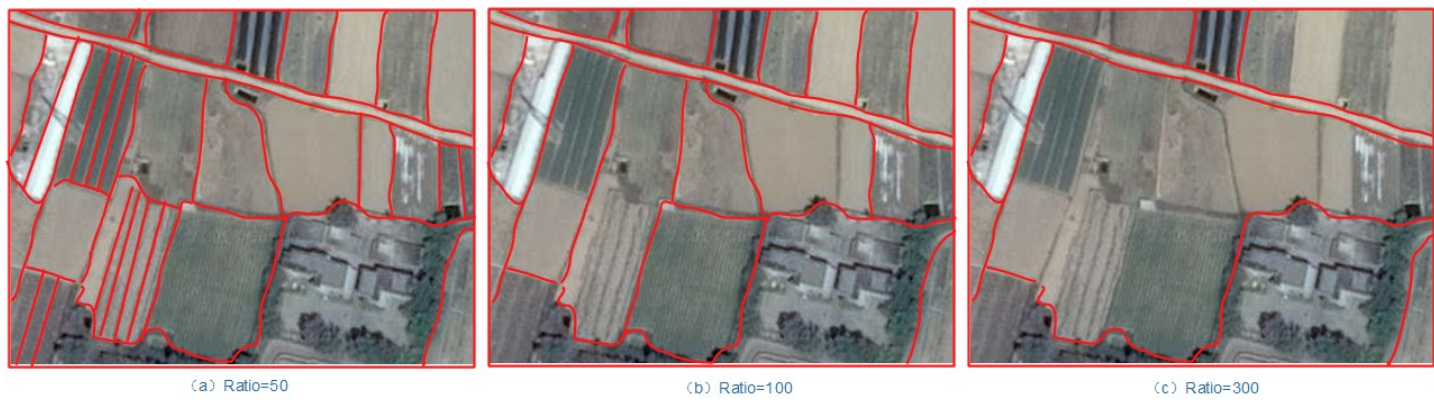

Figure 3. Experimental image segmentation comparison under different experimental conditions.

As can be seen from Fig. 3, different scale sizes have different classification effects. The difference is obvious. This experiment uses three kinds of classifiers for fuzzy recognition classification, that is, Mahalanobis distance, support vector machine and neural network fuzzy. The final classification results are shown in Figure 4. The results of experimental data fuzzy decision fusion experiments show that the fusion classification of
Mahalanobis distance, support vector machine and neural network can improve the overall classification effect, and it can achieve effective judgment for some areas with insignificant modes. (c) The phenomenon of determining buildings and shadows as water bodies has been improved, and effective determination of buildings and bare land has been achieved.

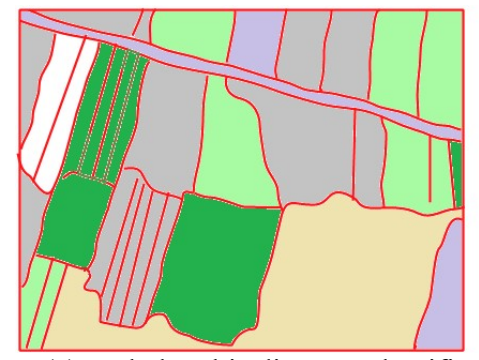

(a) Mahalanobis distance classification

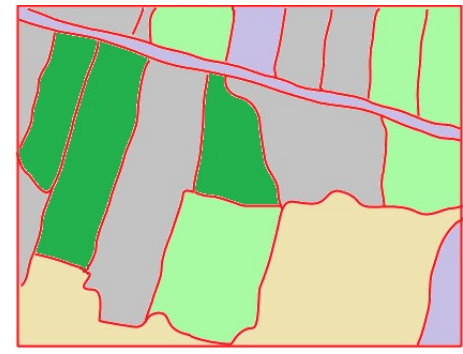

(b) Vector classification

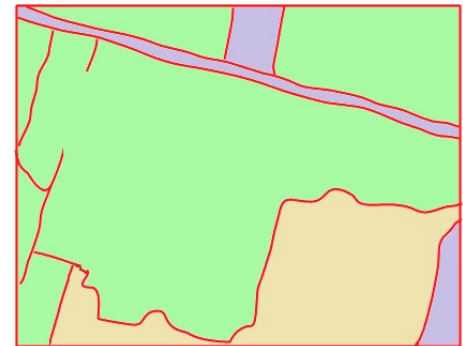

(c) Fuzzy neural classification

Figure 4. Image classification results.

Based on the results of combinatorial classification, the image patches in thematic vector data of historical land use are taken as analysis units. We analyze the composition of each category in each patch. If there is a large proportion of the patch that is inconsistent with the historical attribute, then it is judged to be a change pattern. Through experimental analysis, it is found that the type of land use is not easy to change for the historical attribute of artificial buildings. For agricultural land areas, it is easy to change to other types of regions. For the green frame area identified in Figure 5, its historical attribute is the vegetation cover area, and after the object oriented classification processing, more than $80 \%$ of the images are different from the historical category, so the green area is automatically identified as the changing area. In the area identified in Figure 5, there is a historical attribute for agricultural land. Through the analysis of the category composition in the region, its main components are building and bare land, so it can be determined that the region has changed, and it is automatically determined as a changing area.

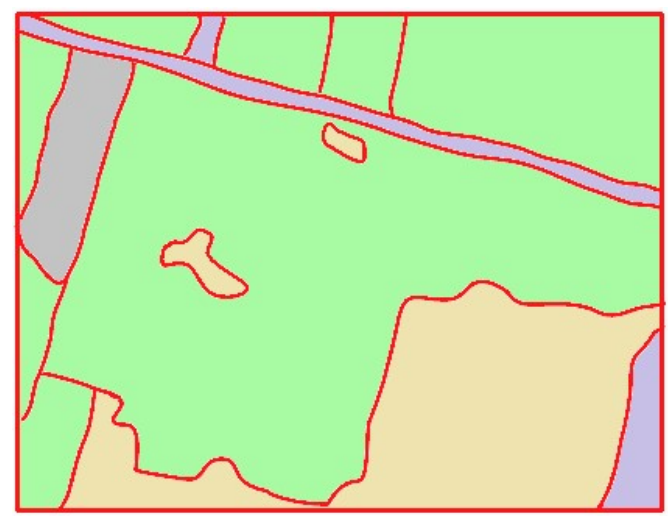

Figure 5. Overlying of change detection results on the original image. 


\section{Conclusions}

The Gis and combined classification methods are used to classify the remote sensing images. The experimental results show that the algorithm can effectively and automatically determine the change phenomenon in the region where the ground object category changes significantly. It is difficult to effectively determine the region with only a small proportion change in the region. Its change. However, this paper proposes a change detection method combining remote sensing image and thematic vector data. The experimental results show that the combined classification method can effectively integrate the advantages of Mahalanobis distance, support vector machine and neural network classification method, and improve the classification accuracy.

\section{References}

1. IM J, JENSEN J R, TULLIS J A. Object-Based Change Detection Using Correlation Image Analysis and Image Segmentation [J]. International Journal of Remote Sensing, 2008, 29 (2): 399-423.
2. FAN Jianrong, Liu Fei, Gong Kuifang, Guo Fenfen, Guo Xiang, Zhang Dingrong. Spatial characteristics of soil erosion in Hengduan Mountain region, eastern Tibet based on GIS [J]. Journal of Arid Land Resources and Environment. 2012, 26 (4): 144-150.

3. Wang Fei, Xing Shihe. Application of GIS and Mathematic Models on Evaluating the Climate Suitability of Land Resources for Sugarcane in Fujian Province [J]. Chinese Journal of Soil Science. 2012, 43 (1): 176-182.

4. Raymond E.E. Jongschaap. Run-time calibration of simulation models by integrating remote sensing estimates of leaf area index and canopy nitrogen [J]. European Journal of Agronomy, 2006, 24 (4): 316-324.

5. GUILHERME L. A. MOTA, RAUL Q. FEOTOSA, HEITOR L. C. COUTINHO, et al. Multi-temporal Fuzzy Classification Model Based on Class Transition Possibilities [J]. ISPRS Journal of Photogrammetry \& Remote Sensing, 2007, 62 (3): 186-200. 